\title{
Commentaries
}

\section{Anticipating the onset of inflammatory bowel disease}

The great interest in identifying susceptibility genes in inflammatory bowel diseases (IBD) has recently triggered the aggregation of family trees throughout Europe and the United States. This has led to a better knowledge of empirical risk and clinical characteristics of familial IBD. One of the most consistent findings in families with Crohn's disease was early onset in most affected children of an affected parent, with an average difference of 15 years. This may be a more general feature of familial IBD, as shown in this issue by Lee et al (see page 808) who observed a similar time span (16-18 years) between generations in 50 pure Crohn's disease, 51 pure ulcerative colitis and 36 mixed disease families.

Genetic anticipation has been suggested as a possible explanation for this finding. ${ }^{1}$ This term denotes a decrease in the age of onset and an increase in severity as a disease is passed through generations. ${ }^{2}$ Genetic anticipation has been described in monogenic neurological illnesses such as Huntington's disease, myotonic dystrophy and more recently Friedreich's ataxia. In these diseases, there is firm evidence that anticipation reflects the effects of genetic factors and has a true molecular basis. Amplification of DNA triplet repeats within or adjacent to the disease gene occurs in successive generations and this DNA instability is associated with increasing disease severity and earlier age of onset. ${ }^{3}$ The list of conditions exhibiting anticipation has grown in the past few years, and recognition of anticipation would be very important for a proper understanding of inherited susceptibility in IBD.

There are difficulties in defining biological anticipation in polygenic disorders such as IBD as the epidemiological data supporting genetic anticipation may be subject to many biases, particularly in retrospective studies. ${ }^{4}$ Increased awareness and diagnostic acuity may influence age at diagnosis in the later generation. In Lee et al's study and in a previous series ${ }^{6}$ there was a shorter time interval between symptom onset and age at diagnosis in children than in parents although the difference only accounted for two years. This ascertainment bias was controlled by Heresbach and colleagues, ${ }^{7}$ who compared the differences in age at diagnosis in second degree relatives and in parent and child. They found a similar inter-generation difference in the second degree relatives (16.5 years) and in first degree relatives (14.2 years) which was consistent with anticipation. Another complicating factor which is inherent in retrospective studies is the differential age at interview, which results in a greater chance of finding a later age at diagnosis in the older generation (recall bias). ${ }^{5} \mathrm{~A}$ third potential bias is the preferential ascertainment of parents with late age at diagnosis. The median age at IBD diagnosis in the affected parents was higher in all published familial series (41 years in Lee et al's study) than that observed in sporadic cases. It might well reflect a selection bias (also called truncation bias) with less recruitment of parents with early onset and reduced fertility. ${ }^{4}$ This bias was displayed in two studies in which parent-child pairs were stratified according to age at diagnosis in the parents. ${ }^{6}{ }^{7} \mathrm{No}$ anticipation persisted in the "early diagnosed" parentchild pairs but did in a Jewish subsample. Finally, the main confounder in these familial studies, which was tackled properly by Lee et al, is the inadequate follow up of the younger generation. Using regression analysis Lee et al showed an inverse correlation between age at diagnosis and calendar year of birth both for patients with familial and non-familial IBD. They thus showed that IBD occurring later in life in the younger generation was not taken into account: the children have not yet lived through their years of susceptibility. This ascertainment bias artificially lowered the mean age of onset of the younger generation. Additional evidence against genetic anticipation was provided by the observation that there was no difference between disease extent (a marker of disease severity) in parent-child pairs. This is consistent with the similarity in clinical characteristics of familial and sporadic IBD. In a recent study no difference was observed between 152 patients with a positive familial history of Crohn's disease and 1164 sporadic cases with regard to the importance of medical treatment and the incidence and extent of excisional surgery. ${ }^{8}$

In summary, assertion of genetic anticipation by mere observation of age at diagnosis and disease severity in complex disorders such as IBD is difficult. ${ }^{9}$ Lee et al's study was itself not without methodological pitfalls such as the use of more than one child per parent. This does not mean that genetic anticipation is definitely excluded. As seen in Huntington's disease, unstable DNA may be present even when the anticipation pattern is similar to regression to the mean, and cases of negative anticipation do occur. ${ }^{10}$ Expansion of the (CAG)n repeat has been reported in Crohn's disease ${ }^{11}$ and in keeping with genetic heterogeneity, subsets of patients (e.g. Jewish) could yet exhibit genetic anticipation. ${ }^{7}$ In clinical practice, the only message available so far for affected parents is that the relative risk of IBD for their children is increased by a factor of 10 to 15 compared with the general population. Presently, we suggest genetic counselling should not be provided about age of onset and disease severity in IBD.

Supported by the Ministère de la Santé et de l'Action Humanitaire (Direction Générale de la Santé) and INSERM (Grant 92/R/2).

J F COLOMBEL D LAHARIE B GRANDBASTIEN

Registre des Maladies Inflammatoires du Tube Digestif du Nord-Ouest de la France (EPIMAD), CHRU Lille, France

1 Polito II JM, Rees RC, Childs B, et al. Preliminary evidence for genetic anticipation in Crohn's disease. Lancet 1996;347:798-800.

2 McInnis MG. Anticipation: an old idea in new genes. Am f Hum Genet 1996;59:973-9.

3 Rosenberg RN. DNA-triplet repeats and neurologic disease. $N$ Engl f Med 1995;350:459-60.

4 Fraser FC. Trinucleotide repeats are not the only cause of anticipation. Lancet 1997;350:459-60.

5 Bayless TM, Picco MF, LaBuda MC. Genetic anticipation in Crohn's disease. Am ₹ Gastroenterol 1998;93:2322-5.

6 Grandbastien B, Peeters M, Franchimont D, et al. Anticipation in familial Crohn's disease. Gut 1998;42:170-4.

7 Heresbach D, Gulwani-Akolkar B, Lesser M, et al. Anticipation in Crohn's disease may be influenced by gender and ethnicity of the transmitting parent. Am f Gastroenterol 1998;93:2368-72.

8 Carbonnel F, Macaigne G, Beaugerie L, et al. Crohn's disease severity in familial and sporadic cases. Gut 1999;44:91-5.

9 Vieland VJ, Huang J. Statistical evaluation of age-at-onset anticipation: a new test and evaluation of its behavior in realistic applications. Am $\mathcal{F}$ Hum Genet 1998;62:1212-27. 
10 Petronis A, Sherrington R, Kennedy JL. Regression to the mean does not exclude anticipation and unstable DNA disease. Am f Hum Genet 1994;55: $589-90$.
11 Cho JH, Fu Y, Pickles M, et al. CAG repeat expansions in subset of families with Crohn's disease [abstract]. Gastroenterology 1997;112:A948.

\section{Intolerance of the dirty intestine}

The mucosal immune system faces the delicate task of co-existence with a luxuriant commensal intestinal bacterial flora $\left(10^{12}\right.$ bacteria/g faeces in the colon, and roughly $10^{3}$ different species, with anaerobes predominating). Yet a protective immune response to invasive enteric pathogens is also mandatory. Of course, any commensal organism can become a pathogen in appropriate circumstances, and the magnitude of this balancing act is illustrated by the similarity between proteins of the harmless commensal Escherichia coli and its pathogenic derivatives (or the Shigella genus). The essential differences between innocent and harmful bacteria reside in toxin production and qualities of adherence to, or penetration of, the intestinal epithelial cell layer.

The barrier function of the intestine is clearly disrupted in active inflammatory bowel disease (IBD), with evidence for greater systemic penetration by commensal organisms. Whether abnormal intestinal permeability precedes intestinal inflammation in individuals predisposed to IBD, is still unclear, although it is a major feature of Crohn's disease relapse. The "passive" intestinal barrier (of the epithelial cell layer linked by junctional complexes and overlaid with mucus) is certainly not (in real life) as fixed as one might suppose. ${ }^{1}$ We constantly face insults to barrier function, either through our own actions (ingestion of non-steroidal anti-inflammatory drugs, alcohol) or from intestinal pathogens. Epithelial cells are also actively involved in defence through chemokine and cytokine release. It is easy to see how a vicious cycle following penetration by luminal bacteria or their products might arise, ending in chronic IBD, yet most of us avoid it.

Over the past six years the study of genetically manipulated rodents has contributed enormously to the understanding of the circumstances which predispose to intestinal inflammation. Ablating the function of a large number of different immunological genes (including interleukin (IL) 2 , IL-10 or $\alpha \mathrm{T}$ cell receptor) or inserting HLA-B27 each independently renders the animal liable to develop spontaneous intestinal inflammation that may usually be attenuated or avoided by breeding and keeping the animals in very clean (SPF) or germ-free facilities. Although genetic loci linked to human IBD have been described, the hunt for the genes themselves continues, so many of the animal genetic abnormalities may be somewhat artificial. Nevertheless, they do provide support, in well defined conditions, for the concept that upsetting the delicate balance between the mucosal immune system, the epithelial cell layer and the commensal bacterial flora results in chronic intestinal inflammation.

Duchmann and coworkers, in this issue (see page 812), have examined the reactivity of $\mathrm{T}$ cell clones, derived from IBD intestinal mucosa, against commensal bacteria. It is clear that the mucosa of active Crohn's disease contains an increased proportion of activated T cells, ${ }^{3}$ and $\mathrm{T}$ cell cloning has generally proved a powerful immunological technique as it provides a culture of $\mathrm{T}$ cells with a single receptor with specificity for short (9-15 amino acid) peptide epitopes. From such clones the major antigenic determinants for helper and cytotoxic $\mathrm{T}$ cells in viral and bacterial infections have been elucidated.

In general, working out the specific antigens to which $\mathrm{CD} 4+\mathrm{T}$ cells in the intestinal mucosa respond is a formidable task, complicated by their very poor antigen specific proliferative responses in culture and the diversity of the luminal antigen mixture. Nevertheless, Duchmann and colleagues have previously presented data that $\mathrm{T}$ cells isolated from the intestinal mucosa of control subjects will proliferate in vitro in response to relatively crude fractions of bacteria isolated from the intestinal (heterologous) flora of a different individual, but not from their own flora. Patients with Crohn's disease, however, have intestinal $\mathrm{T}$ cells capable of responding to their own (autologous) flora. Thus Crohn's disease could be interpreted as a failure of mucosal tolerance to the indigenous flora, an idea in keeping with the data from the animal models and with the clinical effectiveness of faecal stream diversion. Despite the diversity of the human intestinal microflora, there is considerable homology between proteins of related species and common carriage of many species by different individuals, so the differences that bring about responses to the heterologous flora in normal subjects are still unclear.

An important technical issue surrounds the way in which the $\mathrm{T}$ cell clones were derived in this paper. It is difficult to get $\mathrm{T}$ cells from the intestinal mucosa to proliferate well in response to antigen, so to produce clones of identical cells the stimulation process had to be non-specificphytohaemagglutinin (PHA) followed by expansion on irradiated allogenic feeder cells. Although the idea is to obtain representative clones, the responses to bacterial sonicates may not reflect the antigen specificities of the initial $\mathrm{T}$ cells. With this caveat, there are three main results. Firstly, there was considerable cross reactivity in the response of CD4+ clones to anaerobic (Bifidobacterium and Bacteroides) and aerobic enterobacteria. Secondly, the authors show that their previous observation whereby $T$ cells from patients with IBD respond to crude preparations of the autologous flora, can be applied (in some cases) at the level of T cell clones. Thirdly, they analysed which bacterial species within a heterologous mixed isolate could stimulate a $\mathrm{T}$ cell clone from a patient with ulcerative colitis and showed that aerobic enterobacteria were mainly responsible and curiously some colonies of a bacterial species (e.g. $E$ coli) might stimulate this clone whereas others would not.

So, on the one hand, there seems to be cross reactivity in the proliferative responses of $\mathrm{T}$ cell clones from patients with IBD between different bacterial species, and, on the other, the responses to the heterologous flora involve many common aerobic species. Where is the window in which "tolerance" to the autologous flora develops or collapses at the $\mathrm{T}$ cell level? A substantial amount of work will be needed to unravel this question. The beauty of $\mathrm{T}$ cell clones is that specificities to individual protein molecules (or other structural bacterial components) can be determined (if bacterial proteins are first purified), and this could sort out the molecular basis of cross reactivity and detect the window(s) in the autologous/heterologous flora in individual cases. Unfortunately each person is likely to be different because of the diversity of MHC class II in the 
human population which presents antigenic peptide to CD4+ T cells.

Duchmann et al's paper is an important contribution to the evidence that difficulties in handling the commensal microflora are the key to IBD. Yet the mucosal immune system is sophisticated, and in addition to the presence or absence of lamina propria CD4+ $\mathrm{T}$ cells that respond to commensal bacterial determinants (as investigated here), there are many other levels of regulation, including unresponsive $\mathrm{T}$ cells and those which produce downregulatory cytokines but do not proliferate. ${ }^{45}$ The relative contributions of these mechanisms in health and their defects in IBD are still not known.

A J S MACPHERSON K J MALOY

Institute of Experimental Immunology, Universitätsspital,
Schmelzbergstrasse 12, CH 8091 Zürich, Switzerland

Department of Medicine,

GKT School of Medicine,

Bessemer Road,

London SE5 9PF, UK

1 Bjarnason I Macpherson A, Hollander D. Intestinal permeability. Gastroenterology 1995;108:1566-81.

Strober W, Ludviksson BR, Fuss IJ. The pathogenesis of mucosal inflammation in murine models of inflammatory bowel disease and Crohn disease. Ann Intern Med 1998;128:848-56.

3 MacDonald TT, Murch SH. Aetiology and pathogenesis of chronic inflammatory bowel disease. Baillière's Clin Gastroenterol 1994;8:1-34.

4 MacDonald TT. Effector and regulatory lymphoid cells and cytokines in mucosal sites. Curr Top Microbiol Immunol 1999;236:113-35.

5 Khoo UY, Proctor IE, Macpherson AJS. CD4+ T cell downregulation in human intestinal mucosa: evidence for intestinal tolerance to luminal bacterial antigens. F Immunol 1997;158:3626-34.
Several studies have reported significant positive associations between colorectal cancer (CRC) and Barrett's oesophagus. ${ }^{1-5}$ In the initial report, 29 colon tumours, 10 of them malignant, were found in 65 patients with Barrett's oesophagus. ${ }^{1}$ The authors suggested that the probability of detecting a similar number of malignant colorectal lesions in an age matched sample of the general population was less than one in a million. The early studies, however, were often hampered by their choice of a "control" group or the complete absence of one. Bias may also have arisen from the discovery of Barrett's oesophagus during the investigation of patients with gastrointestinal symptoms referable to their presentation of a CRC. In addition, the existence of asymptomatic but prevalent cases of both colon cancer and of Barrett's oesophagus is now more widely recognised than it was a decade ago. ${ }^{6}$ Despite reservations about the validity of these studies, the finding that colon tumours seemed to be more common in patients with oesophageal intestinal metaplasia than in patients with functional bowel symptoms or with positive faecal occult blood tests is interesting on two accounts.

Firstly, the implications for a colorectal cancer screening programme of a positive association between the index cancer and Barrett's oesophagus are considerable. The prevalence of Barrett's oesophagus in the general population has been reported to be as high as $1 \%^{7}$ and initial studies suggested a prevalence in patients with Barrett's oesophagus of $8-45 \%$ for colorectal adenomas and $7-15 \%$ for colorectal carcinomas. ${ }^{3}$ If an association of this degree was definitively established then colorectal screening might be targeted specifically at individuals with confirmed Barrett's oesophagus.

Secondly, if Barrett's oesophagus and colorectal neoplasia are associated, what is its aetiological basis and is there a biological explanation for the apparent relation? Shared environmental factors or genetic predisposition might result in an association but have not been clearly demonstrated. Nevertheless, although there is a lack of experimental data linking the two conditions, they do have some interesting features in common. Increased dietary fat intake and reduced fruit and vegetable consumption has been associated with an increased risk of CRC and possi- bly with Barrett's oesophagus. ${ }^{6}$ In addition there are genetic alterations that are common to both conditions: abnormalities such as p53 gene mutation and allelic loss of chromosome 18q are associated with the progression of the adenoma-carcinoma sequence in human colon and more recently have been identified in Barrett's mucosa but it is unclear whether genetic changes are a cause or consequence of intestinal metaplasia in the oesophagus. ${ }^{89}$ Further insights into a putative genetic association may be gained by studying Carney syndrome, an autosomal dominant multi-system syndrome of which both Barrett's oesophagus and colonic polyps can be features. ${ }^{10}$

It was clear from the early studies that the prevalence of colorectal neoplasia in patients with Barrett's oesophagus was high; if colonoscoped about a third would be found to have either an adenoma or a cancer. ${ }^{3}$ Indeed the largest study using the renowned Mayo clinic database found that $14 \%$ of 175 records with a Barrett's oesophagus diagnosis also contained a colorectal cancer diagnosis. ${ }^{4}$ What was less clear was how much higher these figures were compared with the general population of similar age who were not being colonoscoped. Two groups have compared the figures for patients with Barrett's oesophagus with those from four small US studies of colonoscopy screening of the general population. Howden and Hornung concluded that although there was no increased risk of colorectal adenoma, there was a fivefold increase in risk of cancer which was statistically significant. ${ }^{3}$ In contrast Poorman et al reviewed all the prospective surveys and using the same colonoscopy screening data concluded that the increased risk of cancer was small and not sufficient for screening. ${ }^{11}$

An alternative epidemiological approach is to make the assumption that the development of oesophageal adenocarcinoma is a marker for previous Barrett's oesophagus as well as its most significant consequence and then to use cancer registry data to examine the coincidence of the two cancers. This has now been done in two studies. The first used US cancer registry data to perform a nested case-control study in which the cases were patients with oesophageal adenocarcinoma and the controls patients with oesophageal squamous cell cancer, for which there is no association with colon cancer. ${ }^{5}$ Compared with patients with squamous cell cancer there was a $44 \%$ increased risk of colorectal cancer developing before or after the oesophageal adenocarcinoma in men (odds ratio (OR) $1.44,95 \%$ confidence interval (CI) 1.03-1.72) but a reduced risk in women (OR $0.39,95 \%$ CI $0.19-0.78$ ). In contrast there was no increase in risks of prostate or breast 
cancer for the adenocarcinoma cases, indicating some specificity to these associations. The authors offer no explanation for the sex difference or for examining it other than the propensity of epidemiologists to break relations down by sex!

In the second study (see page 819) Lagergren and Nyren have used the comprehensive Swedish cancer registry to identify all patients with colon cancer registered during 1958-92. Only 11 patients subsequently developed oesophageal adenocarcinoma, giving an overall increased risk of $20 \%$ (standardised incidence ratio 1.2 , 95\% CI $0.6-2.1$ ) with similar risks in men and women. What is striking is that despite having a cohort of 118000 elderly patients accumulating over half a million person years, the expected number of oesophageal adenocarcinomas was only 9.5 emphasising how rare this cancer has been in Sweden. On the basis of recent oesophageal cancer mortality rates in the UK the expected number for a cohort of this size would have been over four times higher. This suggests that the prevalence of Barrett's oesophagus in Sweden over this period was low compared with the UK. It is notable that over the same period there was a substantial increase in oesophageal cancer mortality in the UK $60 \%$ in men and $35 \%$ in women) with an increasing proportion due to adenocarcinoma, whereas in Sweden mortality increased only $11 \%$ in men and fell $40 \%$ in women. ${ }^{12}$ Although the rarity of this cancer in Sweden has reduced the power of their study to exclude small increases in risk there are no good reasons for believing that their findings are unrepresentative of other European countries.

So what is the "take home" message for the jobbing gastroenterologist? Current data are not sufficient to rule out a modest, less than twofold increased risk of colorectal cancer in patients with Barrett's oesophagus or oesopha- geal adenocarcinoma and vice versa. This might be explained by either genetic predisposition or common environmental risk factors. None the less an association of this magnitude is not sufficient by itself to warrant screening and is unlikely to be a source of fruitful research.

RICHARD F A LOGAN MAEVE M SKELLY

Department of Public Health and Epidemiology,

University of Nottingham Medical School,

Queen's Medical Centre,

Clifton Boulevard,

Nottingham NG7 2UH, UK

1 Sontag SJ, Schnell TG, Chejfec G, et al. Barrett's oesophagus and colonic tumours. Lancet 1985;i:946-9.

Robertson DAF, Ayres RCS, Smith CL. Screening for colonic cancer in patients with Barrett's oesophagus. BMF 1989;298:650.

3 Howden CW, Hornung CA. A systematic review of the association between Barrett's esophagus and colon neoplasms. Am f Gastroenterol 1995;90: 1814-19.

4 Limberg PJ, Ahlquist DA, Talley NJ, et al. Risk and site predominance of colorectal cancer with Barrett's oesophagus: a cohort study [abstract]. Gascolorectal cancer with Barrett's

5 Vaughan TL, Kiemeney LALM, McKnight B. Colorectal cancer in patients with esophageal adenocarcinoma. Cancer Epidemiol Biomarkers Prev 1995;4:93-7.

6 Kim R, Weissfeld JL, Reynolds JC, et al. Etiology of Barrett's metaplasia and oesophageal adenocarcinoma. Cancer Epidemiol Biomarkers Prev 1997;6: 369-77.

7 Cameron AJ, Zinsmeister AR, Ballard DJ, et al. Prevalence of columnarlined (Barrett's) oesophagus: comparison of population-based clinical and autopsy findings. Gastroenterology 1990;99:918-22.

$8 \mathrm{Wu}$ T-T, Watanabe T, Heitmiller R, et al. Genetic alterations in Barrett esophagus and adenocarcinomas of the esophagus and esophagogastric junction region. Am F Pathol 1998;153:287-94.

9 Gleeson CM, Sloan JM, McGuigan JA, et al. Barrett's oesophagus: Microsatellite analysis provides evidence to support the proposed metaplasiadysplasia-carcinoma sequence. Genes Chromosomes Cancer 1998;21:4960 .

10 Nwokoro NA, Korytkowski MT, Rose S, et al. Spectrum of malignancy and premalignancy in Carney syndrome. Am f Med Genet 1997;73:369-77.

11 Poorman JC, Lieberman DA, Ippoliti AF, et al. The prevalence of colonic Poorman JC, Lieberman DA, Ippoliti AF, et al. The prevalence of colonic
neoplasia in patients with Barrett's esophagus: prospective assessment in patients 50-80 years old. Am $\mathcal{F}$ Gastroenterol 1997;92:592-6.

12 Cheng KK, Day NE. Oesophageal cancer in Britain. BMF 1992;304:711.
Significant titres of circulating antibodies reacting with a wide range of normal tissue components occur with variable frequency in several liver disorders. Those that are of particular relevance to liver disease include the antinuclear (ANA), smooth muscle (SMA), liver-kidney microsomal (LKM), antimitochondrial (AMA), and antineutrophil cytoplasmic (ANCA) antibodies. In some conditions, such as autoimmune hepatitis (AIH) and primary biliary cirrhosis (PBC), they form part of the diagnostic criteria. ${ }^{12}$ Their presence in others, such as chronic viral hepatitis, may serve to confuse. These autoantibodies are routinely detected by indirect immunofluorescence on sections of appropriate tissues or isolated cells and an experienced eye is required for interpretation of the different patterns of immunofluorescent staining. It has long been recognised, however, that antibodies giving apparently similar staining patterns often react with different antigens in the substrates used for their detection. In order to improve the sensitivity and specificity of these antibodies for different liver disorders, and to provide more objective methods for their detection, considerable efforts have been made in recent years to identify the specific antigens with which they react, with some success. Thus, the cytochrome P450 isoform IID6 has been identified as the target of LKM antibodies that are particularly associated with so called type $2 \mathrm{AIH},{ }^{3}$ whereas the AMA that are most specific for PBC are now known to react with the E2 com- ponents of the mitochondrial 2-oxoacid dehydrogenase complexes. $^{2}$

ANCA, which have been extensively studied in patients with segmental necrotising glomerulonephritis, Wegener's granulomatosis and other vasculitides, are classified into two major subtypes according to whether they give cytoplasmic (cANCA) or perinuclear (pANCA) patterns of immunofluorescent staining on ethanol fixed neutrophils. ${ }^{45}$ cANCA reacting with proteinase 3 are particularly associated with Wegener's granulomatosis and some classic pANCA are specific for myeloperoxidase but both subtypes are notably heterogeneous and a number of other target autoantigens have been identified, including azurocidin, lactoferrin, and cathepsin G. ${ }^{4}$ Additionally, some pANCA ("atypical" or xANCA) give staining patterns that are subtly different from those of classic pANCA, implying reactivities with yet other antigens. ${ }^{5}$

The relevance of pANCA to liver disease relates to the observation that these antibodies occur in more than $80 \%$ of patients with primary sclerosing cholangitis (PSC) ${ }^{6}$ For a time, they were thought to be a potential diagnostic marker of this disease, ${ }^{7}$ but recent studies have indicated that high titres of pANCA also occur with similar frequency in AIH. ${ }^{8}$ These two conditions can usually be differentiated on the basis of histological and cholangiographic findings but they share several autoimmune features which can complicate the diagnosis. If the antigenic specificities of pANCA in PSC and AIH differ, and can be shown to be distinct from pANCA in other conditions, specific tests for these reactivities would be a useful addition to the diagnostic repertoire. Two very 
recent studies suggest that some progress is being made in this regard. The first indicates that atypical pANCA associated with ulcerative colitis, PSC and AIH react with nuclear lamins $\mathrm{A}, \mathrm{C}$ and $\mathrm{B} 1$, and with the lamin $\mathrm{B}$ receptor, and that these reactivities are never observed in systemic vasculitis. ${ }^{9}$

The second report, which appears in this issue (see page 867), provides persuasive evidence that nearly all patients with AIH with classic pANCA have antibodies that react with the non-histone chromosomal proteins HMG1 or HMG2, or both. How these antibodies to HMG relate to pANCA is, however, uncertain. Absorbing the sera with purified HMG only partially abrogated pANCA staining, perhaps because most of the sera also contained antibodies reacting with two other known pANCA targets: cathepsin $\mathrm{G}$ and lactoferrin. Conversely, and somewhat surprisingly, $64 \%$ of PBC sera that were negative for classic pANCA by immunofluorescence (despite having antibodies against cathepsin $G$ or lactoferrin) were positive for anti-HMG. Unfortunately, the authors did not include patients with PSC in their main study group although in the discussion they cite preliminary data indicating that antibodies to HMG may occur much less frequently (two of eight) in pANCA positive patients with PSC. Thus, neither of these two reports provides definitive evidence identifying subspecificities of pANCA that may be useful in the differential diagnosis of hepatobiliary diseases but both provide sufficiently suggestive data to indicate that further studies along these lines in much larger groups of patients with a wider range of liver disorders are worthwhile.

I G MCFARLANE

Institute of Liver Studies,

King's College Hospital,

Denmark Hill, London SE5 9RS, UK

email:ian.mcfarlane@kcl.ac.uk

1 Johnson PJ, McFarlane IG. Meeting report: International autoimmune hepatitis group. Hepatology 1993;18:998-1005.

2 Leung PSC, Coppel RL, Ansari A, et al. Antimitochondrial antibodies in primary biliary cirrhosis. Semin Liver Dis 1997;17:61-9.

3 Manns MP, Griffin KJ, Sullivan KF, et al. LKM-1 autoantibodies recognize a short linear sequence in P450IID6, a cytochrome P-450 monooxygenase. a short linear sequence in P45011

4 Kallenberg CGM, Brouwer E, Weening JJ, et al. Anti-neutrophil cytoplasmic antibodies: Current diagnostic and pathophysiological potential. Kidney Int 1994;46:1-15

5 Semrad CE, Terjung B, Worman HJ. Antineutrophil cytoplasmic and other autoantibodies in primary sclerosing cholangitis. In: Krawitt EL, Wiesner $\mathrm{RH}$, Nishioka M, eds. Autoimmune liver diseases. 2nd edn. Amsterdam: Elsevier, 1998:305-20.

6 Klein R, Eisenburg J, Weber P, et al. Significance and specificity of antibodies to neutrophils detected by western blotting for the serological diagnosis of primary sclerosing cholangitis. Hepatology 1991;14:1147-52.

7 Lo SK, Fleming K, Chapman R. Prevalence of anti-neutrophil antibody in primary sclerosing cholangitis and ulcerative colitis using an alkaline phosphatase technique. Gut 1992;33:1370-5.

8 Targan SR, Landers C, Vidrich A, et al. High-titer antineutrophil cytoplasmic antibodies in type-1 autoimmune hepatitis. Gastroenterology 1995;108: mic antib.

9 Terjung B, Herzog V, Worman HJ, et al. Atypical antineutrophil cytoplasmic antibodies with perinuclear fluorescence in chronic inflammatory bowel diseases and hepatobiliary disorders colocalize with nuclear lamina proteins. Hepatology 1998;28:332-40. 